\title{
Pathway to the Presidency: The Perceived Impact of Identity Structures on the Journey Experiences of Women College Presidents
}

\author{
Gloria Oikelome \\ Reading Area Community College \\ U.S.A.
}

ABSTRACT: This phenomenological study utilizes the framework of Intersectionality to explore the perceived impact of race, gender, and other identity structures on the journey experiences of seven White and six African American women college presidents. Findings suggest that while gender is becoming more peripheral, the interlocking tensions of race and gender often shape the journey experiences of African American women, with race appearing to be a salient factor. Despite challenges resulting from these social constructs, the women employed various strategies for navigating the presidential pipeline including mentorship, leadership development programs, and firm assurance of institutional fit.

KEYWORDS college presidents, women, African-American, leadership identity, intersectionality

\author{
Rationale for Study \\ Method and Analysis \\ Results and Discussion \\ Conclusion \\ Note \\ References \\ Author Contact
}

Viewed as the pinnacle of academic administration, the college presidency is a benchmark of status and achievement in the academy. Yet despite women's achievements in higher education access, success, and progression to faculty and administrative roles, the higher education leadership pipeline has failed to produce the desired outcome of increased representation of women presidents (Cook \& Kim, 2012; Kellerman \& Rhode, 2014). The narrative regarding women college presidents has focused on the remarkable strides they have made in increasing their representation from 9\% of all post-secondary institutions in 1986 to $30 \%$ in 2016 (Cook \& Kim, 2012; Gagliardi, Espinosa, Turk, \& Taylor, 2017). While this assessment rightfully touts the significant development over the years, a more analytical narrative reveals a different and conflicting picture with regard to women of color and the college presidency. Although the number of minority college presidents slowly increased over the last 30 years, women of color remain the most underrepresented in the presidency. In 2011, women of color comprised approximately $9 \%$ of all college presidents across two- and four-year institutions (Cook \& Kim, 2012). In 2016, this number diminished to 5\%, making women of 
color the most underrepresented in the college presidency (Gagliardi et al., 2017). In contrast to an increasingly diverse student demographic, the profile of college and university leaders has hardly changed in more than a quarter century, and there is a general consensus that higher education should better reflect the diverse population it serves in order to prepare students to enter an increasingly globalized and pluralistic society (Cook \& Kim, 2012; Gagliardi et al., 2017; Longman \& Anderson, 2016). Multiple factors, including the impending retirement of aging presidents, the increased turnover and shorter tenures of college presidents, and the varying needs of an increasingly diverse student population, have created a window of opportunity for achieving diversification (Cook \& Kim, 2012).

\section{Rationale for Study}

College presidents traditionally shape the educational philosophy, direction, and culture of their institution (Blumenstyk, 2014). Diversification of the college presidency brings a variety of thought, innovation, and divergent perspectives to address the needs of a dynamic student population and helps colleges navigate the various challenges facing higher education including affordability, decreased funding, changing pedagogy, disruptive technologies, and retention, to name just a few (Gagliardi et al., 2017; Kirwan, 2008). Conversely, a lack of diversity in leadership represents a loss of varying perspectives and voices to inform the decision-making process (Karkouti, 2016; Longman \& Madsen, 2014). Since the 1990s, the higher education student demographic has undergone a dramatic transformation unparalleled in Western higher education (Blumenstyk, 2014). In contrast to its rapidly changing student population, however, the higher education leadership demographic has remained homogenous, and women, particularly minority women college presidents, are significantly underrepresented (Cook \& Kim, 2012; Gagliardi et al., 2017).

A central criticism of the literature on leadership in higher education has been that much of it has focused on the perspectives of older, White males (Amey, 2006; Holmes, 2008). However, some research surrounding the career development, progression, and leadership styles of women in higher education has surfaced (Cress \& Hart, 2009; Dahlvig \& Longman, 2010; Enke, 2014; Longman \& Anderson, 2016; Patitu \& Hinton, 2003; Tunheim \& Goldschmidt, 2013). Specifically, various studies have emerged on the progression and strategies for success for women college presidents (Brown, 2005; Madsen, 2006). However, Holmes (2008) maintained that much of this research classifies women presidents as a singular group without taking into consideration the potential impact of race or the potentially differing experiences of minority women, specifically African American women. Additionally, much of the research on gender places White women as the universal female subject, while studies on race place African American men as the universal racial subject. As a result, the experiences of African American women become invisible in the discourse (Crenshaw, 1989; Wilder, Jones, \& Osborne-Lampkin, 2013). Jackson and Harris (2007) noted that 
the limited number of African American women presidents poses a challenge for conducting studies.

The literature is replete with studies on the need for diversification in the academy (Holmes, 2008; Jackson \& Harris, 2007; Madsen, 2006). However, few studies focus simultaneously on African American and White women's perceptions of the impact of race and gender on their journey experiences as college presidents. In one study, Settles, Pratt-Hyatt, and Buchanan (2008) explored the similarities and differences in African American and White women's perceptions of their gender. Utilizing a qualitative focus group methodology, they conducted six different focus groups in which 14 African American and 17 White women of varying ages, socioeconomic backgrounds, and professions participated. They found that African American and White women both experience sexism, harassment, or gender-based discrimination in the workplace; however, White women participants perceived more benefits of gender compared to their African American counterparts. Investigating African American women presidents in relation to other groups, specifically White women, may lead to a deeper understanding and additional insight into strategies for success. Such an exploration also presents an opportunity not only to examine the impact of gender, race, and other identity structures separately but also to acknowledge the confluence and intersection of race and gender as experienced by African American women.

Intersectionality is a framework used to explore how the socially and culturally constructed categories of race, gender, and class interact dependently to create a system of oppression (Hamilton-Mason, Hall, \& Everett, 2009). The intersections are referred to in various terms including the race-gender-class matrix, the intersectional paradigm, interlocking systems of oppression, multiple axes of inequality, and the intersectional approach (Berger \& Guidroz, 2010). While the initial components of race, gender, and class have traditionally formed the tripartite construct used to understand the lives of African American women, researchers have broadened intersectionality to encompass the relationship among other dimensions, including sexuality, religion, education, immigration status, occupation, socioeconomic status, and age, thereby allowing intersectionality to be more applicable to various groups (Jones, Misra, \& McCurley, 2013). Intersectional analysis allows for the exploration of deep biases based on race, class, and gender that are still pervasive in our culture and philosophy. As the next generation stands poised to take the reins of higher education leadership, insight into the perceived impact of race, gender, and other identity structures on the journey experiences of women, including ethnic minority women, who have successfully navigated to the presidency will be valuable for expanding the pipeline of potential women college presidents. The aim of the study described in this article was not to focus on a Black/White binary paradigm. Instead, given the national focus on moving diversity agendas forward in colleges and universities and creating inclusive campus environments, this research intended to advance an effective understanding of the journey experiences of women college presidents from all ethnic backgrounds. 


\section{Method and Analysis}

This qualitative study employed a phenomenological approach to give voice to, explore, and compare how White and African American women college presidents made meaning of and perceived the impact of race, gender, and other identity structures on their journey experiences to college presidency. A phenomenological approach was appropriate for this study for several reasons. First, the study is concerned with attempting to understand experiences from the participant's point of view. Secondly, it provides an understanding of subjective experience and allows insight into the motivations, actions, and shared experiences of the participants. Thirdly, phenomenology focuses on what participants experienced and how they experienced it.

The study was guided by three questions:

1. What are the experiences of African American and White women in their journey to the college presidency?

2. How do African American and White women perceive the impact of race, gender, and other salient identity structures on their journey experiences to the college presidency?

3. What strategies and approaches can be identified from the experiences of African American and White Women for successfully navigating the presidential pipeline and advancing to the college presidency?

\section{Setting and Participants}

This study employed purposive sampling, a non-probability technique to obtain participants. Purposive sampling provides the opportunity to focus on the particular characteristics of a population. This enables the best responses to the research questions (Barbour, 2001). The sample included women college presidents, either African American or White, who have served or currently serve at four-year institutions ${ }^{1}$. The focus on four-year institutions is reflective of the higher representation of women at two-year institutions. In 2011, approximately $33 \%$ of presidents at two-year institutions were women, compared to $26 \%$ at fouryear institutions (Cook, 2012). Twenty potential participants were identified by searching the websites of four-year institutions on the East Coast of the United States as well as following up on suggestions from professional colleagues. Overall, 13 women college presidents, six African American and seven White, agreed to participate. Each participant completed a consent form and agreed to be recorded as per the conditions of the IRB. To ensure anonymity, pseudonyms were created for each participant and identifiable information about their respective institutions was omitted. Table 1 provides a demographic profile of the participants. The identity table was constructed based on the participant's self-ascribed 
identities. When answering the question "How do you identify?" participants were asked to present their identity structures in the order that each considered to be foremost. For all the participants but one, race or gender was selected as the foremost identity structure, followed by either religion, nationality or sexual orientation.

Table

Demographic Profile of Participants

\begin{tabular}{cccccc}
\hline Participant & Identity Structures & Pathway & Institution & Tenure & Positions \\
\hline President A & Woman, White, Catholic & Academic & Private & $23 \mathrm{yrs}$ & 4 \\
President B & Woman, African American & Academic & Public & $3 \mathrm{yrs}$ & 6 \\
President C & Woman, White, Lesbian & Practitioner & Public & $5 \mathrm{yrs}$ & 5 \\
President D & African American Woman & Academic & Public & $14 \mathrm{yrs}$ & 4 \\
President E & Woman, White, Lesbian & Academic & Public & $1 \mathrm{yr}$ & 4 \\
President F & African American, Woman & Academic & Public & $2 \mathrm{yrs}$ & 7 \\
President G & White, Woman, Catholic & Academic & Private & $10 \mathrm{yrs}$ & 4 \\
President H & Woman, Lesbian, White & Academic & Public & $8 \mathrm{mos}$ & 4 \\
President I & White Woman & Academic & Private & $14 \mathrm{yrs}$ & 6 \\
President J & Woman, African American & Academic & Private & $2 \mathrm{yrs}$ & 5 \\
President K & American Woman & Academic & Public & $7 \mathrm{yrs}$ & 5 \\
President L & Woman, African American & Academic & Public & $13 \mathrm{yrs}$ & 4 \\
President M & Woman, White, Gay & Academic & Public & $1 \mathrm{yr}$ & 4 \\
\hline
\end{tabular}

\section{Data Collection and Analysis}

Data were collected from semi-structured, one-on-one interviews consisting of 12 open-ended questions. Each interview ranged from 40 minutes to approximately 75 minutes and provided a discovery-oriented method of exploring the experiences and perspectives of research participants (Guion, Diehl, \& McDonald, 2001). The semi-structured format provided flexibility and the ability to guide, revise and probe deeper with questions in real time as new information emerged. While the schedule of questions remained consistent in each interview, there was ample opportunity to engage with participants individually and use probes to encourage further elaboration on their answers. As the individual personality or response style of the participants became apparent, the interview questions were adjusted as necessary to evoke responses that were meaningful and salient to each participant. Field notes were used to record observed verbal and non-verbal behaviors during interviews.

The interviews were transcribed and each participant was provided with a resulting transcript to ensure accuracy and integrity of the responds. Analysis of 
the data was iterative and conducted in several phases. The first phase involved listening again to each original recording but this time reading along with the completed and respondent-validated transcript. This process allowed for a more holistic and intuitive understanding of what was being said about the phenomenon. The next stage of analysis involved organizing the data by utilizing NVivo, a comprehensive computer-assisted qualitative data analysis software, used to manage, shape, and create themes from unstructured information. Two approaches were utilized in the coding process. The first involved the creation of pre-constructed headings and labels and the subsequent coding of transcript data into the existing headings and labels. The second included a "bottom-up" approach in which transcripts were reviewed and codes created as they arose in the data (Denzin \& Lincoln, 2005). The resulting themes were consolidated to form conceptual similarities, and subsequently a single detailed narrative was developed around each theme. Multiple iterations of the coding process resulted in the identification of various conceptual similarities, which were streamlined and consolidated to form three central themes. Subsequently, a single detailed narrative was developed around each theme.

\section{Results and Discussion}

Three overarching themes emerged from the analysis of the narratives: challenges to progression, competence supersedes identity, and strategies for navigating the pipeline. While the themes may not necessarily reflect the experience of each woman president, as a prototype case of a White or African American women president does not exist, they represent the overarching areas of commonalities in the participants' experiences.

\section{Challenges to Progression}

The challenges to progression that the participants indicated fell into two areas: challenges stemming from identity structures and challenges stemming from organizational structures. Challenges stemming from identity structures included gender, race, and sexual orientation. Race, gender, and other identity structures are socially constructed, and how each woman experienced or perceived the impact of these structures is shaped by various factors. With regard to gender bias, there was a wide spectrum in how the women perceived the impact of gender on their progression. Some indicated bias during graduate school, most noted gender bias as faculty while pursuing promotion and tenure, and others recalled experiences of bias once they had advanced into administrative roles as well as in the presidential selection process. All but one of the 13 women indicated experiences of gender bias at various points in their progression. For some the bias was subtle; for others it was overt. Although all the women in the study had successfully attained presidencies, those who perceived the impact of gender bias 
noted that its impact on their progression was in terms of the time it took to progress and advance to the next level and the emotional toll it took on them during their progression.

We are talking about moving the needle because as women for years we are still only about $26 \%$ of presidents in this country and even though the percentage of women who earn doctorates has gone up, in terms of opportunities there is a glass ceiling and we also say if you break that glass ceiling, make sure that the shards of glass don't kill you. (President C)

When they were asked to identify instances of gender bias and challenges, almost all of the African American women shifted to speaking about racial bias concurrently, and all were resigned to the fact that various undertones of racial bias would continue to exist in the academy. The women related experiences of marginalization, lack of support, and exclusion from informal networks as faculty striving to advance through the rank and file in departments where they were the only person of color. It was frequently the case that they referred to their race as a whole, whether male or female, and the overall dearth of minority representation among tenured faculty and administrative leaders, and the continued politics of race in the academy. Although half of the African American women were specific in identifying first as women before identifying as "Black women," the sobering reality in some instances was, as one participant aptly stated:

The world sees you as a woman of color and I think what that does is when you are in a position of leadership it makes insecure males more combative. So I think that even though we women of color don't see, I mean I don't see the world through this I'm a woman of color, the world sees you as a woman of color. (President D)

The inability to decipher which identity structure was in operation depicts the lived realities of intersectionality in the experiences of African American women. This difficulty in separating gender and race is the foundation for intersectionality. The findings suggest that the lived realities of intersectionality, specifically the interplay of race and gender, are potent in the narratives of the African American women. This assertion is supported in much of literature on African American women and progression in the academy; in short, race still matters (Brown, 2005; Diggs, Garrison-Wade, Estrada, \& Galindo, 2009; Griffin, Bennett, \& Harris, 2011; Hinton, 2010; Holmes, 2008; Jackson \& Harris, 2007). This study also supports the assertion that, in some instances, attempts to examine the two identities of race and gender separately pose a challenge (Crenshaw, 1989; Zamani, 2003). This was evidenced when the African American women narrated their experiences of gender bias, but almost immediately began providing examples of racial bias as well.

The findings suggest that for African American women, race may still be imposed externally as a salient factor, even when the individual chooses not confer salience to her racial identity. For some of the women interviewed, the lack of other minorities to inspire them or serve as role models for information or guidance led to feelings of isolation and marginalization. Additionally, the African American 
women in this study perceived that social perceptions and expectations appear to confer more stringent standards of performance on them as compared to their White counterparts. As one participant noted:

One thing about Black women, you always have to prove you are qualified and White people, they are born to lead and that is the way this society operates. (President L)

In the narrative of each African American woman, there was a sense of resignation that continued social constructs and challenges stemming from racial discrimination, whether real or perceived, operate as salient factors in the progression of African American women in the academy. Following an interview for a presidential role, one African American participant explained that the feedback she received from the consultant was really a "code" that those applying for presidencies, particularly African American women or women in general, have come to recognize.

The consultant said 'they are really interested in you' and then this is the code word I hear, 'they are really concerned how the donors will perceive you,' or we can't see you in front of the legislators, can't see the legislators listening to you. So that's the code for presidents because donors are disproportionately, White business men. They are looking at the package I am presenting and deciding that oh no, I can't have her playing golf, she's not going to fit in there, can't have her drinking scotch with the guys. (President $\mathrm{J}$ )

One unexpected finding involved the identity structure of sexual orientation as indicated by four of the 13 women presidents. All four women were White with three self-identified as lesbian and one self-identified as gay. While race and gender were central to this study, the intersectional framework informing this research allowed for other potential identity structures to be incorporated as the participants chose. In this instance, the identity structure of sexual orientation emerged from these participants. Given the small number of openly gay college presidents, this finding was unexpected but equally worthy of discussion. In the same manner in which the African American women seemed resigned to challenges stemming from race and the race-gender matrix, the four women acknowledged that there will always be societal challenges stemming from sexual identity. However, these sexually minority women did not perceive their sexual identity as a barrier or challenge to their advancement in the academy. It should be noted that all of the four women who identified as lesbian or gay also identified as White and each was resolute in her assertion and perception that her sexual identity had no bearing on her progression.

Well the gay piece, this has been such a non-issue, I cannot believe how much of a non-issue it is. It is just phenomenal to me that we go there so quickly in 2015 that it doesn't seem to impact anyone in anyway. (President E)

Analysis of the responses of the White and African American women with regard to bias stemming from various identity structures reveals that while all of 
the White women in the study indicated other identity structures in addition to race, there were no instances in which the White women described challenges they faced as a "White lesbian" or a "White Catholic." Additionally, in the case of the four women who identified as lesbian, greater emphasis was placed on challenges they may have experienced as women but not as lesbians. In this study, the experience of bias due to interlocking identities is limited to the African American women as they described their experiences as "Black women." These findings suggest that there is a salience to race in presidential attainment that may not exist for other identity structures. Additionally, White women did not express the same resignation regarding gender bias that African Americans expressed regarding race. Although the women personally did not identify one identity structure as more salient than another, the narratives of some of the African American women indicate that race continues to be perpetrated as a salient factor in progression even when individuals choose not to focus on it. This is difficult to assert, given that the women all eventually succeeded in their progression and attained presidencies; however, their journey experiences were not without challenges stemming from racial identity.

In addition to challenges stemming from identity structures, the findings of this study indicate that women also face challenges resulting from organizational structure and culture. Regardless of identity structure, each participant related some experience of how some aspects of the organizational structure and culture of the academy posed a barrier for women in advancing into leadership roles. Twelve of the 13 presidents progressed through some variation of the traditional academic route (progression from faculty to academic administrative roles such as dean or provost/vice president). Although each indicated that she could see the benefit of recruiting presidents from other areas in the institution, they all affirmed that the traditional academic pathway would probably always remain the most desired progression for presidents. These findings are consistent with those in national studies (Cook \& Kim, 2012) and in the general literature (Brown, 2005; Jackson \& Harris, 2007; Madsen, 2006; Turner, 2007), which indicate that the traditional academic route is the most prevalent for women college presidents.

The single participant who had a student affairs background discussed the particular challenges of not having the traditional academic background. The literature provides evidence of the challenges women, in particular, face if they are coming from non-academic backgrounds (Cook, 2012). The organizational structure and culture of the academy still favors the traditional academic background. In those instances where there might be flexibility in the background of a potential candidate, the findings suggest that women perceive the traditional academic structure and background to be more beneficial to men. Current research on college presidents indicate that there are more male college presidents from non-academic or non-traditional backgrounds compared to female college presidents.(Cook \& Kim, 2012; Gagliardi et al., 2017). 


\section{Competence Supersedes Identity}

The second theme that emerged in the study is the notion that competence supersedes identity. While each participant acknowledged and articulated various experiences stemming from their gender, racial, sexual, or interconnecting factors, there was a consensus among all the participants with respect to the desire to be defined beyond their various identities, choosing rather to focus on and describe the repertoire of skills, expertise, and proficiency they acquired with each level of advancement, all of which culminated into achieving the pinnacle of their career, a college presidency.

I think what is tipping here is and I think this is exactly right and gives me great hope for the future is that, competency trumps identity, competence, capacity, will, grit, the ability to deliver outcomes, again to get the job done that you have been hired to do trumps my identity, your identity. (President C)

What was remarkable in the narratives of these women is that regardless of what others perceived of their identities, they believed that their success in achieving a college presidency ultimately came down to their competence, capability, and ability to lead. At the heart of the acknowledgement that gender, racial, and sexual identity bias still exist, there was a palpable confidence exuding from each woman as she described the qualities and abilities that she believed led to her attaining a presidency. The array of skills included strategic planning, financial acumen, emotional intelligence, authenticity in leadership, and the ability to be change agents.

The second theme that emerged in the study is the notion that competence supersedes identity. While each participant acknowledged and articulated various experiences stemming from their gender, racial, sexual, or interconnecting factors, there was a consensus among all the participants with respect to the desire to be defined beyond their various identities, choosing rather to focus on and describe the repertoire of skills, expertise, and proficiency they acquired with each level of advancement, all of which culminated into achieving the pinnacle of their career, a college presidency. President $\mathrm{C}$ summed up this sentiment as follows:

I think what is tipping here is and I think this is exactly right and gives me great hope for the future is that, competency trumps identity, competence, capacity, will, grit, the ability to deliver outcomes, again to get the job done that you have been hired to do trumps my identity, your identity. (President C)

The narratives of these women is remarkably consistent in that regardless of what others perceived of their identities they believed that their success in achieving a college presidency ultimately came down to their competence, capability, and ability to lead. Each woman described the various experiences that allowed them to enhance their proficiency, build capacity for leadership, and 
develop their repertoire of skills. The array of skills included strategic planning, financial acumen, emotional intelligence, authenticity in leadership, and the ability to be change agents. In the current environment of declining enrollment, increasing tuition, diminished funding, and increased call for accountability, the effective president must be able to strategically articulate and advocate for the mission of the institution. Financial acumen, including the ability to build relationships with various constituents in order to raise funds was seen as an essential skill. Other women expressed that a college president required the ability to incorporate emotional intelligence into thinking and decision-making, particularly as they deal with so many different constituents on a daily basis. All of the women embraced transformational and authentic leadership. The ability to be present, authentic, and honest was critical. Being something other than your authentic self was viewed as too stressful. Finally, each woman viewed herself as a change agent, not only when she became a president, but also when they were leading in each role prior to a presidency. The ability to be a change agent resulted in greater opportunities which subsequently led to advancement.

At the heart of the acknowledgement that gender, racial, and sexual identity bias still exits, there was a palpable confidence exuding from each woman. President $\mathrm{F}$ believed that her leadership qualities and abilities led to attaining a presidency.

They didn't hire me because of my St. John's suit, and I am convinced of that. You need to be absolutely prepared to understand all the dynamics of running an institution. You can fight about color, you can fight about gender, but you can't find about being strategic. It's about what you bring to the table. They are hiring you because they want to know what you're going to bring as a change agent or a progress agent to that institution (President F)

President $L$ similarly articulated her confidence by stating, "I think the world is starting to figure out that it's not so much about your gender or race; it's your leadership skills." She went on to say that once an institution figures this out, it may even come as a surprise to them:

Sometimes campuses are surprised that women are the best candidate and offer the best skills set and they are surprised themselves when they hear themselves saying, we want to hire that woman or that person of color, we think they will be a good fit for us (President $L$ ).

In summary, by relating their various expertise and skills, each of 13 women presidents affirmed that her achievement of a presidential role resulted from competence and competence superseded identity in her case.

\section{Strategies for Navigating the Pipeline}

The final theme includes the various strategies and approaches the women incorporated in navigating the presidential pipeline and achieving success. Regardless of identity, there was a general consensus that the strategies of 
mentorship, seizing opportunities, leadership development, and understanding institutional fit were some of the essential approaches for any women aspiring to a presidential role. Mentorship, both formal and informal, was the most common strategy for navigating the pipeline. Mentors were essential in providing encouragement, advice, insight, guidance, and, in some instances, sponsorship. The participants were mentored by both men and women; however, the African American women were more likely to be mentored by men, specifically White men. While other studies provide strong evidence on the benefits of mentorship, they are not conclusive about the effects of same gender or cross-gender mentoring. Instead there is evidence to show that diversity in mentoring not only increased women's visibility and access to resources; it enhanced their psychosocial, career, and personal development needs (Brown, 2005; Turner, 2007). Brown (2005) explained that the prevalence of male mentors may be invariably linked to the greater number of males in executive higher education leadership positions.

Having benefitted from some form of mentoring, each woman was active in mentoring others who aspire into various leadership roles in higher education, including a presidential role. Other studies cite mentoring relationships, taking additional opportunities, and participation in leadership programs as common strategies for aspiring women leaders (Brown, 2005; Holmes, 2004; Jackson \& Harris, 2007; Madsen, 2006; Turner, 2007).

A second strategy for navigating the pipeline was participation in leadership development. Nine of the 13 women presidents had participated in one or more formal leadership development program. The programs are intentionally geared for those who are considering a presidency or ready to begin the process of applying for these positions. For some of the women, participating in a leadership program was the impetus for applying for presidential positions. Others found the networking and opportunity to engage with others who are considering a presidency valuable. Four of the 13 women chose not to participate in a leadership program because they felt they had other significant opportunities to experience leadership. Two of the women were appointed into interim positions, and they felt that experience afforded them more insight into a presidency than a leadership program could. The findings revealed that participation in these formal leadership programs was invariably linked to mentorship, as the women were selected to participate in these programs and sponsored by mentors.

The ability to seek and take on additional opportunities was another strategy for navigating the pipeline. Aspiring women were encouraged to step into unfamiliar areas within the institution, volunteer for various services, serve on committees, and participate and present at conferences. Taking on opportunities may not always be convenient, but the benefits are important for progression and advancement. Aspiring women were also encouraged to actively seek such opportunities and put themselves forth for various initiatives and opportunities, rather than spending time "waiting in the wings" to be discovered.

Finally, a key strategy that emerged in the findings was that of understanding institutional fit. In the discussions surrounding the underrepresentation of women and minority women in the college presidency, 
much of literature ascribes the burden to aspects of organizational structure and culture, lack of mentorship or sponsorship, lack of exposure to various areas and opportunities to acquire essential skills, and the tendency of governing boards as gatekeepers to choose candidates that mirror them (Brown, 2005; Jackson \& Harris, 2007; Madsen, 2006; Turner, 2007). While these reasons are strongly supported and well documented, they do not speak to the finding in this study regarding the importance of understanding institutional fit. "Institutional fit" as described by the women presidents in this study involves being strategic in incorporating skills and abilities, acquiring critical information, and matching characteristics, attributes, and identity, so as to ensure the right fit.

Understanding institutional fit is not only knowing where the institution is now but having insight into where it is headed. Having insight into institutional fit can be challenging as the concept is often taught by experience. In some cases, a candidate may experience failure but the lessons learned can be valuable for the next attempt. Of the 13 women presidents, five were successful in gaining a presidential position on their first attempt. The remaining eight had one or more failed attempts, with the most being four. Some of the perceived reasons for failure stemmed from bias due to various identity structures, while other reasons simply came down to institutional fit. The findings revealed that it was not always easy for the women to decipher the precise reason. In some instances, they "just knew" when there was evidence of bias, even when it was very subtle. In those instances, they were confident that the institutional fit was appropriate yet they were still unsuccessful. In other instances, the women did not perceive any bias and expressed that there may have just been insider information that they were not privy to. Having that insider information is also an essential part of institutional fit, and the women encouraged aspiring candidates to be diligent in asking questions and gathering as much information as possible. For all of the women, institutional fit was still invariably linked to identity, as most of the women intentionally sought out places where they were confident their various identity structures would be embraced. These women indicated that they were unwilling to mask their identity or restrain their personality or style in order to fit into a particular organizational culture. As a result, they were intentional in selecting those institutions at which they were confident their authenticity would not suffer.

\section{Conclusion}

This study exploring the journey experiences of White and African American women presidents provides an understanding of their journey to the presidency and the perceived impact of race, gender, and other identity structures on their progression. From an analysis of the narratives of the participants, it is apparent that preparation for higher education leadership does not occur serendipitously. There are challenges along the way, including challenges stemming from identity structures and organizational structures. While the participants in this study acknowledged that there may always be some level of bias predicated on their 
identities, they were able to provide examples to support their assertion that, for them, competence superseded identity. Additionally, the women provided examples of the various strategies they employed in navigating the pipeline and achieving presidential appointments. The conclusion of the participants that competence superseded identity does not eliminate the importance of fostering diversity and representation in higher education leadership; rather, the women were intentional in highlighting their belief that acquiring and succeeding in a role as critical as college president requires specific skill sets regardless of background.

The findings of this study suggest that women in academy continue to be impacted by socially constructed gender roles in the very environment that is touted as an arena for understanding and fostering diversity. If White women are characteristically similar to their male counterparts on those elements that would distinguish a candidate as viable for a presidency, what does this imply for their continued underrepresentation? Although the influence of gender by itself appears to be more and more peripheral, gender still matters. Similarly, if African American women are characteristically similar to their White counterparts on those elements that would distinguish a candidate as viable for a presidency, what does this imply for their continued underrepresentation? While the findings indicate that African American women are unwilling to be deterred by racial bias in their aspiration and attainment of a presidency, the intersecting oppressions of gender and race are so historically rooted to their experiences that race still matters. In the framework of intersectional analysis, the aim of this study was not to debate whether individuals with multiple subordinate group identities (e.g., African American women) are more disadvantaged than those with single subordinate identities (e.g., White women). Rather, the study simply attempts to highlight how the forms of oppression experienced by those with multiple, intersectional, disadvantaged identities differ from the forms of oppression experienced by people with a single disadvantaged identity. Promoting an understanding of varying experiences and perspectives is critical to higher education success and to the strengthening of our democracy (Kellerman \& Rhode, 2014).

The constantly changing higher education environment demands leaders who are visionary and collaborative. As higher education prepares the next generation of leadership, it will be interesting to observe how upcoming generations of women of all backgrounds respond to the challenges facing women in the academy and how the current historical and social movements influence the pathway experiences of future women presidents. Attainment of a college presidency is a remarkable but challenging achievement. For the women presidents in this study, different types of challenges were more significant than others due to conflicting societal norms; however, each woman showed that it is possible to overcome challenges in order to achieve her goal.

This study provided insight into the perceived impact of race, gender, and other identity structures on the journey experiences of White and African American women presidents; however, there are additional areas for exploration. One area for future consideration is the intersecting factors of race and sexual orientation. In this study, African American women presidents reported challenges stemming 
from both racial and gender bias. As the same time, White women identifying as lesbian or gay indicated that this identity structure did not impact their attainment of presidential roles. A potential study is to explore the progression of African American women in higher education who identify as lesbians to determine the salient factors in their experiences. Such a study may provide additional insight into the salience of race. Given the low number of openly LGBTQ presidents and the limited research on this topic, this study could be extended to both men and women of all racial backgrounds to gain insight into how the intersection of gender, race, and sexuality or that of gender and sexuality shapes the experiences of college presidents.

Another area for future study is related to diversity in mentorship relationships, including gender diversity or racial diversity. African American women presidents in this study indicated that most of their mentors were White men, while the White women presidents indicated that they had been mentored by women. An investigation on the impact and differences in cross-gender, samegender and cross-race mentoring for women presidents could provide insight into whether different mentoring needs exist for various racial groups and whether there are any particular advantages or disadvantages in mentorship diversity.

\section{Note}

1. This study does not aim to present a Black/White binary of the experiences of women college presidents. While African American women have typically been the focus in discussions of minority women college presidents, women of other ethnic backgrounds are moving into presidential roles (Gagliardi et al., 2017). Analyzing the journey experiences of other ethnic minority women may provide insight into the potential challenge that all ethnic minority women face in achieving college presidencies.

\section{References}

Amey, M. J. (2006). Leadership in higher education. Change: The Magazine of Higher Learning, 38(6), 55-58.

Barbour, R. S. (2001). Checklists for improving rigor in qualitative research: A case of the tail wagging the dog? British Medical Journal, 322, 1115-1117.

Berger, M. T., \& Guidroz, K. (Eds.). (2010). The intersectional approach: Transforming the academy through race, class, and gender. Chapel Hill, NC: University of North Carolina Press. 
Blumenstyk, G. (2014). American higher education in crisis?: What everyone needs to know. New York, NY: Oxford University Press.

Brown, T. M. (2005). Mentorship and the female college president. Sex Roles. 52 (9-10), 659-666. doi:10.1007/s1119-005-3733-7

Cook, B., \& Kim, Y. (2012). The American college president 2012. Washington, DC: American Council on Education.

Cook, S. G. (2012). Women presidents: Now $26.4 \%$ but still underrepresented. Women in Higher Education, 21(5), 1-3.

Crenshaw, K. (1989). Demarginalizing the intersection of race and sex: A Black critique of antidiscrimination doctrine, feminist theory and antiracist politics. University of Chicago Legal Forum, 1989, Article 8.

Cress, C. M., \& Hart, J. (2009). Playing soccer on the football field: The persistence of gender inequities for women faculty. Equity \& Excellence in Education, 42(4), 473-488.

Dahlvig, J. E., \& Longman, K. A. (2010). Women's leadership development: A study of defining moments. Christian Higher Education, 9(3), 238-258.

Denzin, N. K., \& Lincoln, Y. S. (2005). The Sage handbook of qualitative research. ( $3^{\text {rd }}$ ed.). Thousand Oaks, CA: Sage.

Diggs, G. A., Garrison-Wade, D. F., Estrada, D., \& Galindo, R. (2009). Smiling faces and colored spaces: The experiences of faculty of color pursing tenure in the academy. The Urban Review, 41(4), 312-333.

Enke, K. A. (2014). Identities, intentionality and institutional fit: Perceptions of senior women administrators at liberal arts colleges in the Upper Midwestern USA. Higher Education Research \& Development, 33(1), 100113. doi:10.1080/07294360.2013.864614

Gagliardi, J. S., Espinosa, L. L., Turk, J. M., \& Taylor, M. (2017) American college president study 2017. Washington, DC: American Council on Education.

Griffin, K. A., Bennett, J. C., \& Harris, J. (2011). Analyzing gender differences in Black faculty marginalization through a sequential mixed-methods design. New Directions for Institutional Research, 151, 45-61.

Guion, L. A., Diehl, D. C., \& McDonald, D. (2001). Conducting an in-depth interview. University of Florida Cooperative Extension Service. Gainesville, FL: Institute of Food and Agricultural Sciences.

Hamilton-Mason, J., Hall, J. C., \& Everett, J. E. (2009). And some of us are braver: Stress and coping among African American women. Journal of Human Behavior in the Social Environment, 19(5), 463-482.

Hinton, D. (2010). Creating community on the margins: The successful African American female academician. Urban Review, 42(5), 394-402. doi:10.1007/s11256-009-0140-3 
Holmes, S. L. (2008). Narrated voices of African American women in academe. Journal of Thought, 43(3-4), 101-124.

Holmes, S. L. (2004). An overview of African American college presidents: A game of two steps forward, one step backward and standing still. The Journal of Negro Education, 73(1), 21-39.

Jackson, S., Harris, S. (2007). African American female college and university presidents: Experiences and perceptions of barriers to the presidency. Journal of Women in Educational Leadership, 5(2), 119-137. Retrieved from http://digitalcommons.unl.edu/cgi/viewcontent.cgi?article $=1000$ \&context=jwel

Jones, K. C., Misra, J., \& McCurley, K. (2013). Intersectionality in sociology. Retrieved from http://www.socwomen.org/wp-content/uploads/swsfact sheet_intersectionality.pdf

Kellerman, B., \& Rhode, D. L. (2014). Women at the top: The pipeline reconsidered. In Longman, K. \& Madsen, S.R. (Eds.), Women and leadership in higher education. Charlotte, NC: Information Age Publishing.

Kirwan, W. E. (2008). Diversifying the American college presidency. Washington, DC: American Council on Education.

Longman \& Madsen (2014). Women and leadership in higher education. Charlotte, NC: Information Age Publishing.

Longman, K. A., \& Anderson, P. S. (2016). Women in leadership: The future of Christian higher education. Christian Higher Education, 15(1/2), 24-37.

Madsen, S. R. (2006). Women university presidents: Career paths and educational backgrounds. Academic Leadership Online. Retrieved from http://works.bepress.com/susan_madsen/18

Karkouti, M. (2016). Professional leadership practices and diversity issues in the U.S. higher education system: A research synthesis. Education, 136(4), 405-412.

Patitu, C. L., \& Hinton, K. G. (2003). The experiences of African American women faculty and administrators in higher education: Has anything changed?. New Directions for Student Services, 104, 79-93.

Settles, I. H., Pratt- Hyatt, J. S., \& Buchanan, N. T. (2008). Through the lens of race: Black and White women's perceptions of womanhood. Psychology of Women Quarterly, 32(4), 454-468.

Tunheim, K. A., \& Goldschmidt, A. N. (2013). Exploring the role of calling in the professional journeys of college presidents. Journal of Leadership, Accountability \& Ethics, 10(4), 30-40.

Turner, C. S. V. (2007). Pathways to the presidency: Biographical sketches of women of color firsts. Harvard Educational Review, 77(1), 1 -38.

Wilder, J., Jones, T. B., \& Osborne-Lampkin, L. T. (2013). A profile of Black women 
in the 21st century academy: Still learning from the "outsiderwithin." Journal of Research Initiatives, 1(1), 27-38.

Zamani, E. M. (2003). African American women in higher education. New Directions for Student Services, 104, 5-18.

\section{Author Contact}

Gloria Oikelome: goikelome@racc.edu

Reading Area Community College, 10 S 2nd St, Reading, PA 19602 